\title{
Light curve analysis of variable stars using Fourier decomposition and principal component analysis
}

\author{
S. Deb ${ }^{1}$ and H. P. Singh ${ }^{1,2}$ \\ 1 Department of Physics \& Astrophysics, University of Delhi, Delhi 110007, India \\ e-mail: sdeb@physics.du.ac.in,hpsingh@physics.du.ac.in \\ 2 CRAL-Observatoire de Lyon, CNRS UMR 142, 69561 Saint-Genis Laval, France
}

Received 8 July 2009 / Accepted 1 September 2009

\begin{abstract}
Context. Ongoing and future surveys of variable stars will require new techniques to analyse their light curves as well as to tag objects according to their variability class in an automated way.

Aims. We show the use of principal component analysis (PCA) and Fourier decomposition (FD) method as tools for variable star light curve analysis and compare their relative performance in studying the changes in the light curve structures of pulsating Cepheids and in the classification of variable stars.

Methods. We have calculated the Fourier parameters of 17606 light curves of a variety of variables, e.g., RR Lyraes, Cepheids, Mira Variables and extrinsic variables for our analysis. We have also performed PCA on the same database of light curves. The inputs to the PCA are the 100 values of the magnitudes for each of these 17606 light curves in the database interpolated between phase 0 to 1 . Unlike some previous studies, Fourier coefficients are not used as input to the PCA.

Results. We show that in general, the first few principal components (PCs) are enough to reconstruct the original light curves compared to the FD method where 2 to 3 times more parameters are required to satisfactorily reconstruct the light curves. The computation of the required number of Fourier parameters on average needs 20 times more CPU time than the computation of the required number of PCs. Therefore, PCA does have some advantages over the FD method in analysing the variable stars in a larger database. However, in some cases, particularly in finding the resonances in fundamental mode (FU) Cepheids, the PCA results show no distinct advantages over the FD method. We also demonstrate that the PCA technique can be used to classify variables into different variability classes in an automated, unsupervised way, a feature that has immense potential for larger databases in the future.
\end{abstract}

Key words. methods: data analysis - stars: binaries: eclipsing - stars: variables: RR Lyraes - stars: variables: Cepheids methods: statistical

\section{Introduction}

The recent interest in the structure and properties of light curves of variable stars has increased because of the large amount of observational data from variable star projects like OGLE (optical gravitational lensing experiment), MACHO (massive compact halo object), ASAS (All Sky automated survey) and NSVS (Northern Sky variability survey). In addition, new techniques to tag variable objects expected in huge numbers from satellite missions like CoRoT (Convection Rotation and Planetary Transits), Kepler, and Gaia in a robust and automated manner are being explored (Debosscher et al. 2007; Sarro et al. 2009). The Fourier decomposition technique is a reliable and efficient way of describing the structure of light curves of variable stars. Schaltenbrand \& Tammann (1971) derived UBV light curve parameters for 323 galactic Cepheids by Fourier analysis. The first systematic use of the Fourier technique was made by Simon (1979) to analyze the observed light variations and radial velocity variation of AI Velorum. The first-order amplitudes and phases from the Fourier fits were then compared with those obtained from linear adiabatic pulsation models to obtain the mass of AI Vel. Simon \& Lee (1981) made the first attempt to reconstruct the light curves of Cepheid variables using Fourier decomposition and to describe the Hertzsprung progression in Cepheid light curves. The method has been applied extensively by various authors for light curve reconstruction, mode discrimination and classification of pulsating stars (Antonello et al. 1986; Mantegazza \& Poretti 1992; Hendry et al. 1999; Poretti 2001; Ngeow et al. 2003; Moskalik \& Poretti 2003; Jin et al. 2004; Tanvir et al. 2005). However, Fourier decomposition by itself is not perfectly suitable for classification of variable stars in large databases as the method works only for individual stars, but it can be used as a preprocessor for other automated schemes (Kanbur et al. 2002; Kanbur \& Mariani 2004; Sarro et al. 2009).

Principal component analysis transforms the original data set of variables with an orthogonal transformation to a new set of uncorrelated variables or principal components. The technique amounts to a straightforward rotation from the original axes to the new ones and the principal components are derived in decreasing order of importance (Singh et al. 1998). The first few components thus account for most of the variation in the original data (Chatfield \& Collins 1980; Murtagh \& Heck 1987). The technique has been used for stellar spectral classification (Murtagh \& Heck 1987; Storrie-Lombardi et al. 1994; 
Singh et al. 1998), QSO spectra (Francis et al. 1992) and for galaxy spectra (Sodré \& Cuevas 1994; Connolly et al. 1995; Lahav et al. 1996; Folkes et al. 1996). There have been a number of studies on the use of PCA in analyzing Cepheid light curves (Kanbur et al. 2002) and RR Lyrae light curves (Kanbur \& Mariani 2004). In both these studies, the input data to the principal component analysis (PCA) are the Fourier coefficients rather than the light curves themselves. Nevertheless, it was noted that PCA was able to reproduce the light curves with about half the number of parameters (PCs) needed by the Fourier technique. In PCA, the first few PCs are usually examined as they contain most of the information about the data.

The PCA has been applied to the light curves of Cepheid variable stars by Kanbur et al. (2002) and RR Lyrae stars by Kanbur \& Mariani (2004). They concluded that PCA is more efficient than the FD method in bringing out changes in the light curve structure of these variables. In our opinion, there is no advantage in the way the PCA was applied because the Fourier coefficients were used as input to the PCA which are themselves the information-bearing coefficients of the light curve structure. Therefore PCA will not extract any additional information except the dimensionality reduction to a few orders. In the case of databases where a variety of variables is present, the method of application of PCA on Fourier coefficients is further complicated by the fact that the optimal order of fit to different light curves is different. When using Fourier coefficients as input to the PCA one has to decide where to make a cut in the Fourier fitting orders. For Fourier decomposition of FU Cepheids, one needs precise Fourier components up to order $\sim 10-15$ in explaining the Cepheid bump progression whereas RR Lyraes need fewer Fourier components $(\sim 2-7)$ to completely describe the light curve structure. Also, if the phase coverage is not smooth, then fitting such light curves with higher orders of the fit may give rise to false bumps that are not associated with the true light curve structures. Therefore it is not meaningful to use Fourier coefficients as input to the PCA when light curves of a large number of variable stars having different variability classes are to be analysed. We demonstrate this fact with the following example:

Suppose a larger database of stars contains RRab, RRc and FU Cepheid variables. The RRc stars are always fitted with a lower order of the Fourier fit as compared to RRab and FU Cepheids. Generally RRc stars need $\sim 2-5$ orders of the fit because of the sinusoidal and symmetric nature of their light curves, RRab 3-7 orders of fit because of their asymmetric light curve, whereas some of the FU Cepheids need to be fitted with $\sim 10-15$ orders of the fit to explain the bump progression. Therefore for FU Cepheids, if the light curves are fitted with fewer orders, the bump progression will not be fitted properly and one will miss the important bump feature. On the other hand, if all the light curves are fitted with higher orders of the fit then one is basically fitting the noise in the case of RR Lyrae stars which will also be reflected in the PCA.

One of the most important advantages of PCA over the FD method is that in PCA, all the light curve data can be processed and analysed in one go if all the phased light curve data can be made of similar dimensions, as we shall demonstrate later, whereas in the FD method each light curve has to be fitted with the optimal order of the fit and analysed individually. This is a very time consuming process for large databases. Therefore, the decision regarding the cut in the order of the fit is manual and hence very cumbersome. Unlike FD, one can decide where to make a cut in the PCA order in light curve reconstruction for all the light curves simply by looking at the cumulative percentages of variance in the data set. The optimal data compression using PCA is enormous, a fact that is quite relevant with the larger databases of the future.

PCA has also the advantage of preferential removal of noise from the light curve data and isolating the bogus light curves, whereas for precise Fourier decomposition, one needs very welldefined and accurate light curves free from noisy, scattered data points and having a good phase coverage. The most significant PCs contain those features which are most strongly correlated in many of the light curves. Therefore, the noise that is uncorrelated with any other features will be represented in the less significant components. Also, by retaining only the most significant PCs to represent the light curves we achieve a data compression that preferentially removes the noise. PCA can be used to filter out bogus features in the data as it is sensitive to the relative frequency of occurrence of features in the data set (Bailer-Jones et al. 1998). However, one distinct disadvantage of PCA is that addition of a single light curve in the analysis requires the entire PCA to be redone.

In this paper, we show the use of PCA directly on the light curve data of more than 17000 stars (RR Lyraes, Cepheids, Eclipsing binaries and Mira variables) taken from the literature and different existing databases. We also apply the FD method to these light curves to determine the Fourier parameters. Denoising should be carried out before the Fourier decomposition if the light curves are noisy. However, the photometric error in the light curves in the case of the present selected database is very small, i.e., the light curve data have a good photometric accuracy $(\sim 0.006-0.14 \mathrm{mag}$ in the case of the OGLE database and $\sim 0.02-0.220 \mathrm{mag}$ in the case of the ASAS database). To investigate the noise in the light curves, we have calculated the unit-lag auto-correlation function on the residual light curves. The auto-correlations are found to be $\ll 1$. Therefore no denoising has been carried out. However, in some light curves there were outliers present. To remove these outliers, we used a robust multi-pass non linear fitting algorithm in IDL (Interactive Data Language). We use light curves (magnitudes at different epochs) as input to PCA and compare the relative performance of the ability of PCA in finding resonances in Cepheids and in the classification of different types of variables as compared to the FD method. We have, therefore, performed independent automated Fourier analysis of all the data sets described in the paper using a computer code developed by us.

Another aim of this paper is to analyze the performance of PCA as a fast, automated and unsupervised classification tool for variable stars. Since one of the important aspects of this paper is to do a preliminary PCA based classification in an unsupervised way on a larger sets of astronomical data, we explore the possibility of its use for future databases. PCA can be used for preliminary classification of variable stars such as classification between pulsating stars and eclipsing binaries and different variability classes.

We present the Fourier decomposition technique using the Levenberg-Marquardt algorithm for non-linear least square fitting (Press et al. 1992) in Sect. 2. We also describe the unit-lag auto-correlation function for finding the optimal order of the fit. Section 3 describes the PCA for dimensionality reduction and light curve reconstruction. Section 4 describes the results obtained by the FD and PCA techniques when applied to study the structure of Cepheid light curves. In addition, we compare the relative performance of FD and PCA for classification of various variability classes in the database selected for the present analysis. In Sect. 5, we present the important conclusions of the study. 
Table 1. Data selected for the present analysis.

\begin{tabular}{lccc}
\hline \hline Data & References & No. of stars selected & Data set \\
\hline RR Lyrae & & 5835 & \\
$I$ band (LMC RRab) & Soszyński et al. (2003) & IA \\
$I$ band (LMC RRc) & Soszyński et al. (2003) & 1751 & IB \\
\hline Fundamental Cepheids & Soszyński et al.(2008) & 1804 & IIA \\
$I$ band (LMC) & Martin et al. (1979) & 6 & IIB \\
$V$ band (LMC) & Moffett et al. (1998) & $13+6$ & IIC \\
$V$ band (LMC+SMC) & & & 1228 \\
\hline Overtone Cepheids & Soszyński et al. (2008) & III \\
$I$ band (LMC) & & 2878 & IV \\
\hline Mira Variables & http://archive.princeton.edu/ asas/ & \\
$V$ band & & 2681 & VA \\
\hline Eclipsing Binary & Wyrzykowski et al. (2003) & 1404 & VB \\
$I$ band (LMC) & Wyrzykowski et al. (2004) & & \\
\hline band (SMC) & &
\end{tabular}

\section{Fourier decomposition technique}

Since the light curves of the selected ensemble of variable stars are periodic, they can be written as a sum of cosine and sine series:

$m(t)=A_{0}+\sum_{i=1}^{N} a_{i} \cos \left(i \omega\left(t-t_{0}\right)\right)+\sum_{i=1}^{N} b_{i} \sin \left(i \omega\left(t-t_{0}\right)\right)$,

where $m(t)$ is the observed magnitude at time $t, A_{0}$ is the mean magnitude, $a_{i}, b_{i}$ are the amplitude components of $(i-1)^{\text {th }}$ harmonic, $P$ is the period of the star in days, $\omega=2 \pi / P$ is the angular frequency, and $N$ is the order of the fit. $t_{0}$ is the epoch of maximum light. Obviously, Eq. (1) has $2 N+1$ unknown parameters which require at least the same number of data points to solve for these parameters. Equivalently, we can write Eq. (1) as

$m(t)=A_{0}+\sum_{i=1}^{N} A_{i} \cos \left(i \omega\left(t-t_{0}\right)+\phi_{i}\right)$

where $A_{i}=\sqrt{a_{i}{ }^{2}+b_{i}{ }^{2}}$ and $\tan \phi_{i}=-b_{i} / a_{i}$. Since period is known from the respective databases, the observation time can be folded into phase $(\Phi)$ as (cf. Ngeow et al. 2003)

$\Phi=\frac{\left(t-t_{0}\right)}{P}-\operatorname{Int}\left(\frac{t-t_{0}}{P}\right)$.

The value of $\Phi$ is from 0 to 1 , corresponding to a full cycle of pulsation and Int denotes the integer part of the quantity. Hence, Eqs. (1) and (2) can be written as (Schaltenbrand \& Tammann 1971)

$m(t)=A_{0}+\sum_{i=1}^{N} a_{i} \cos (2 \pi i \Phi(t))+\sum_{i=1}^{N} b_{i} \sin (2 \pi i \Phi(t))$,

$m(t)=A_{0}+\sum_{i=1}^{N} A_{i} \cos \left[2 \pi i \Phi(t)+\phi_{i}\right]$

with relative Fourier parameters as

$R_{i 1}=\frac{A_{i}}{A_{1}} ; \phi_{i 1}=\phi_{i}-i \phi_{1}$

where $i>1$. The combination of coefficients $R_{i 1}, \phi_{i 1}$ where $i=$ $2,3,4 \ldots$ can be used to describe the progression of the light curve shape in the case of Cepheids and other variables and can be used for variable star classification. In Table 1, we list all the variable star light curve data that has been subjected to the analysis. In the case of the data taken from the OGLE database (Soszyński et al. 2003, 2008; Wyrzykowski et al. 2003, 2004), the number of stars seems to be more than the actual number presented in the database. This is because of the fact that we have not tried to remove the overlapping stars in different OGLE fields as this will not affect our analysis. In the case of data from Martin et al. (1979), the stars with poor phase coverage have been left out.

The estimation of the optimal number of terms to be used in the Fourier decomposition of the individual light curve is not straightforward. As has been pointed out by Petersen (1986), if $N$ is chosen too small, a larger number of Fourier parameters can be calculated from a given observation and the resulting parameters will have systematic deviations from the best estimate. On the other hand, if $N$ is chosen too large, we are fitting the noise. Following Baart (1982), Petersen (1986) adopted the calculation of the unit-lag auto-correlation of the sequence of the residuals in order to decide the right $N$ so that the residuals consist of noise only. It as defined as

$\rho:=\frac{\sum_{j=1}^{n}\left(v_{j}-\bar{v}\right)\left(v_{j+1}-\bar{v}\right)}{\sum_{j=1}^{n}\left(v_{j}-\bar{v}\right)^{2}}$,

where $v_{j}$ is the $j$ th residual, $\bar{v}$ is the average of the residuals and $j=1, \ldots n$ are the number of data points of a light curve. The value of $v$ is basically the residuals of the fitted light curve

$v=m(t)-\left[A_{0}+\sum_{i=1}^{N} A_{i} \cos \left(2 \pi i \Phi(t)+\phi_{i}\right)\right]$.

It should be noted that for the calculation of $\rho$ we must choose the ordering of $v_{j}$ given by increasing phase values rather than the ordering given by the original sequence. A definite trend in the residuals will result in a value of $\rho$ equal to 1 , while uncorrelated residuals give smaller values of $\rho$. In the idealized case of residuals of equal magnitude with alternating sign, $\rho$ will be approximately equal to -1 . A suitable value of $\rho$ can be chosen using Baart's condition. According to this, a value of $\rho \geq[n-1]^{-1 / 2}$ (where $n$ is the number of observations) is an indication that it is likely that a trend is present, whereas a value of $\rho \leq[2(n-1)]^{-1 / 2}$ indicates that it is unlikely that a trend is present. Baart therefore used the following auto-correlation cut-off tolerance

$\rho_{\mathrm{c}}=\rho($ cut $)=[2(n-1)]^{-1 / 2}$.

While computing the Fourier parameters of all the light curve data selected for the present analysis we have taken care that 

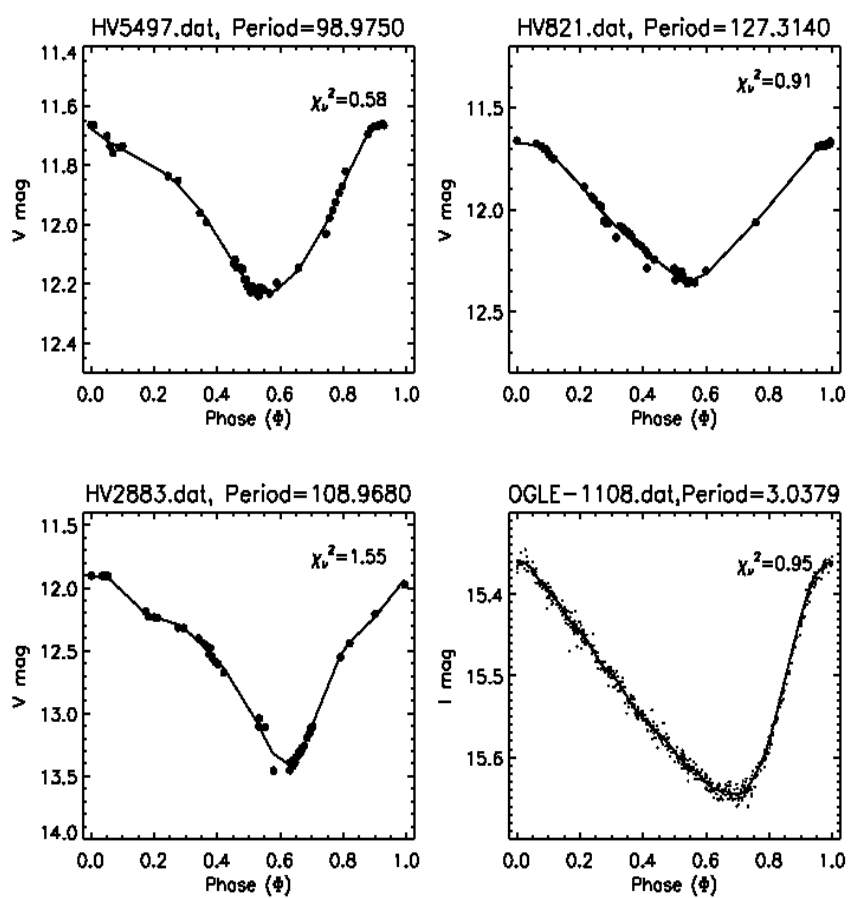

Fig. 1. Fitted light curves for fundamental mode long period Cepheids from Moffett et al. (1998).

Baart's condition is satisfied. The optimal order of the fit for RRc, RRab, FU Cepheids (OGLE), first overtone (FO) Cepheids, eclipsing binaries and Mira variables are 3, 5, 12, 10, 4 and 4 respectively. The longer period data for FU Cepheids from Martin et al. (1979) and Moffett et al. (1998) are fitted with the fifth order of the fit because of the relatively small numbers of data points. A typical example of a fitted light curve of all types of variables with the optimal order of the fit is shown in Fig. 2.

All the data sets in Table 1 are finally fitted with the optimal order of the fit and the fitted light curves are used to derive the Fourier phase and amplitude parameters from the Fourier coefficients. Figure 1 shows the fitted light curves of FU Cepheids. Although the number of data points for the longer periods are less, the phase coverage is sufficient to do the Fourier decomposition. Although the phase coverage is poor, the fits are reasonably good. The lower right panel shows an example of a short period fundamental mode Cepheid from the OGLE-III database which has a good phase coverage. $\chi_{v}^{2}$ is the chi square per degree of freedom $(v)$ of the fit. The degree of freedom $(v)$ is the number of data points minus the number of parameters of the fit. The Fourier decomposition parameters $\left(a_{i}, b_{i}\right)$ for Cepheids have been computed based on the optimal order of the fit by the calculation of the unit-lag auto-correlation function

\section{Principal component analysis}

The principal component analysis transforms the original set of $p$ variables by an orthogonal transformation to a new set of uncorrelated variables or principal components (PCs). It involves a simple rotation from the original axes to the new ones resulting in principal components in decreasing order of importance. The first few $q$ components $(q \ll p)$ usually contain most of the variation in the original data (Chatfield \& Collins 1980; Murtagh \& Heck 1987). This feature of the PCA has been used in astronomical data analysis primarily for the purpose of reducing the dimensionality of the data and as a preprocessor for other
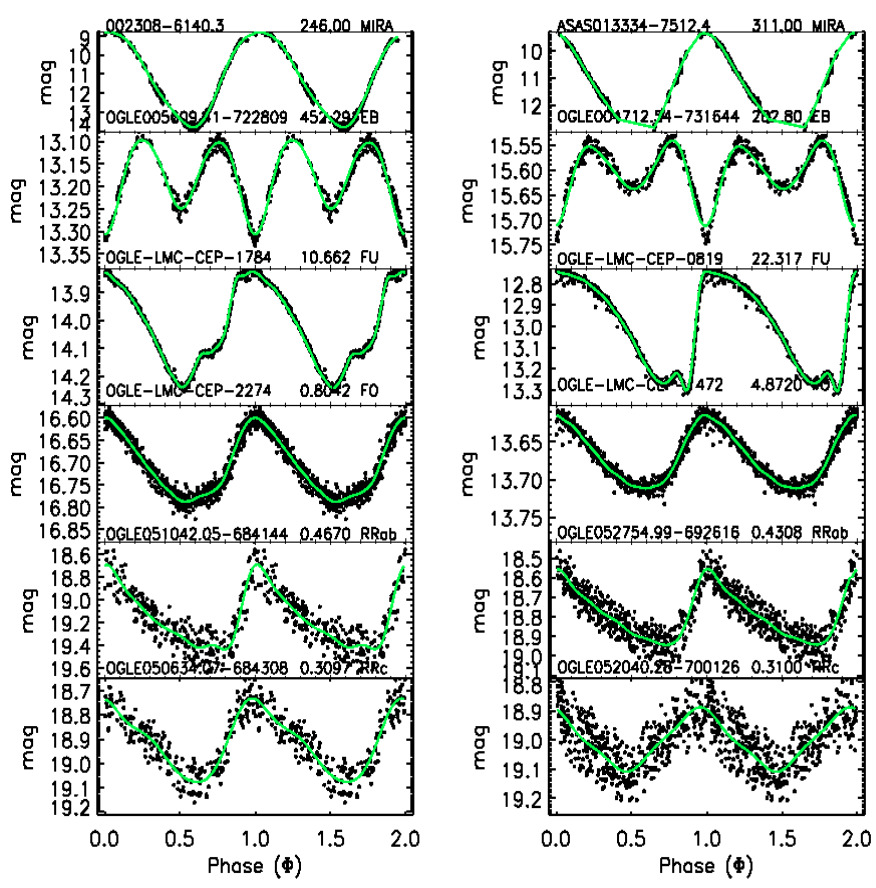

Fig. 2. Fitted light curves of different classes of variables used in the analysis obtained with the optimal order of the fit. The caption at the top of each panel shows the variable name, period and type of variable respectively. We have RR Lyrae variables (RRc, RRab), Cepheid variables (Fundamental mode (FU) and first overtone (FO)), eclipsing binaries (EB) and Mira variables (MIRA).

automated techniques like artificial neural networks (ANN). The application of PCA to the light curve analysis of variable stars has been limited to a few studies (Hendry et al. 1999; Kanbur et al. 2002, 2004; Tanvir et al. 2005). In the following, we briefly describe the transformation.

Let $m_{i j}$ be the $p$ magnitudes corresponding to $n$ light curves. Let us define the $n \times p$ matrix by $\mathbf{X}=x_{i j}$,

$x_{i j}=\frac{m_{i j}-\bar{m}}{s_{j} \sqrt{n}}$

with

$\overline{m_{j}}=\frac{1}{n} \sum_{i=1}^{n} m_{i j}$

and

$s_{j}^{2}=\frac{1}{n} \sum_{i=1}^{n}\left(m_{i j}-\overline{m_{j}}\right)^{2}$

where $\overline{m_{j}}$ is the mean value and $s_{j}$ is the standard deviation. Using such standardization we find the principal components from the correlation matrix (cf. Murtagh \& Heck 1987)

$C_{j k}=\sum_{i=1}^{n} x_{i j} x_{j k}=\frac{1}{n} \sum_{i=1}^{n}\left(m_{i j}-\overline{m_{j}}\right)\left(m_{i k}-\overline{m_{k}}\right) /\left(s_{j} s_{k}\right)$,

with the axis of maximum variance being the largest eigenvector $\boldsymbol{e}_{1}$ associated with the largest eigenvalue $\lambda_{1}$ of the equation

$C \boldsymbol{e}_{1}=\lambda_{1} \boldsymbol{e}_{1}$.

The next (second) axis is to be orthogonal to the first and another solution of Eq. (8) gives the second largest eigenvalue $\lambda_{2}$ and the 
corresponding eigenvector or the principal component $\boldsymbol{e}_{2}$. Hence the proportion of the total variation accounted for by the $j^{\text {th }}$ component is $\lambda_{j} / p$, where $p$ is also the sum of the eigenvalues (Singh et al. 1998).

Let us suppose that the first $q$ principal components are sufficient to retain the information on the original $p$ variables. Therefore, we now have a $(p \times q)$ matrix $\mathbf{E}_{q}$ of eigenvectors. The projection vector $\boldsymbol{Z}$ onto the $q$ principal components can be found by

$Z=x \mathbf{E}_{q}$,

where $\boldsymbol{x}$ is vector of magnitudes defined by

$x_{i j} s_{j} \sqrt{n}+\overline{m_{j}}=m_{i j}$,

and can be represented by

$\boldsymbol{x}=\boldsymbol{Z \mathbf { E } _ { q } ^ { \mathrm { T } }}$.

We obtain the final light curve $\boldsymbol{x}_{\text {rec }}$ by multiplying $x$ by $s_{j} \sqrt{n}$ and adding the mean. $Z$ is a $(n \times q)$ matrix and $\mathbf{E}_{q}{ }^{\mathrm{T}}$ is a $(q \times p)$ matrix and hence the reconstructed light curve is the original $(n \times p)$ matrix.

With the phase $(\Phi)$ as the epoch for each light curve available from Eq. (3), we interpolate and obtain 100 mag for phase 0 to 1 in steps of 0.01 . Therefore, each light curve now consists of 100 data points (magnitudes) normalized to unity. The input to the PCA are these 100 points of magnitudes for each of the light curves. We also emphasize that while applying PCA to the phased magnitudes of light curves, Fourier coefficients are not used to interpolate the light curves. We have used standard interpolation routines in IDL to generate interpolated magnitudes in a light curve. Two examples of the result of interpolation are shown in Fig. 3. There are 223 actual data points for the Mira variable (lower panel) and 100 interpolated magnitudes have been obtained.

\section{Analysis of light curves}

In the following analysis, we compare the capabilities of FD and PCA for structural analysis of Cepheids and the classification accuracy for different classes of variable stars.

\subsection{Structural analysis and classification}

\subsubsection{Fundamental mode (FU) Cepheids}

We use the light curve data for 1829 FU classical Cepheids from various sources as mentioned in Table 1 (Data set IIA+IIB+IIC). The majority of the data used in the analysis are from the OGLE database. The Fourier decomposition of all the 1829 Cepheid light curves was independently done by us for the calculation of the Fourier decomposition parameters as described in Sect. 2. We verified that all the Cepheid light curves selected in the present study give a satisfactory light curve shape with no numerical bumps or deviations when reconstructed using the Fourier parameters.

PCA is performed on an input matrix consisting of a $1829 \times$ 100 array corresponding to $100 \mathrm{mag}$ from phase 0 to 1 for 1829 FU Cepheids. The result of the PCA output is shown in Table 2 . We see that first 10 PCs contain nearly 90 percent of the variance in the data. Figure 4 shows the reconstruction of four FU Cepheid light curves using the first 1, 3, 7 and 10 PCs.

Kanbur et al. (2002) have tried to explain the resonances using the PCA on the Fourier coefficients $\left(a_{i}, b_{i}\right)$. But due to the

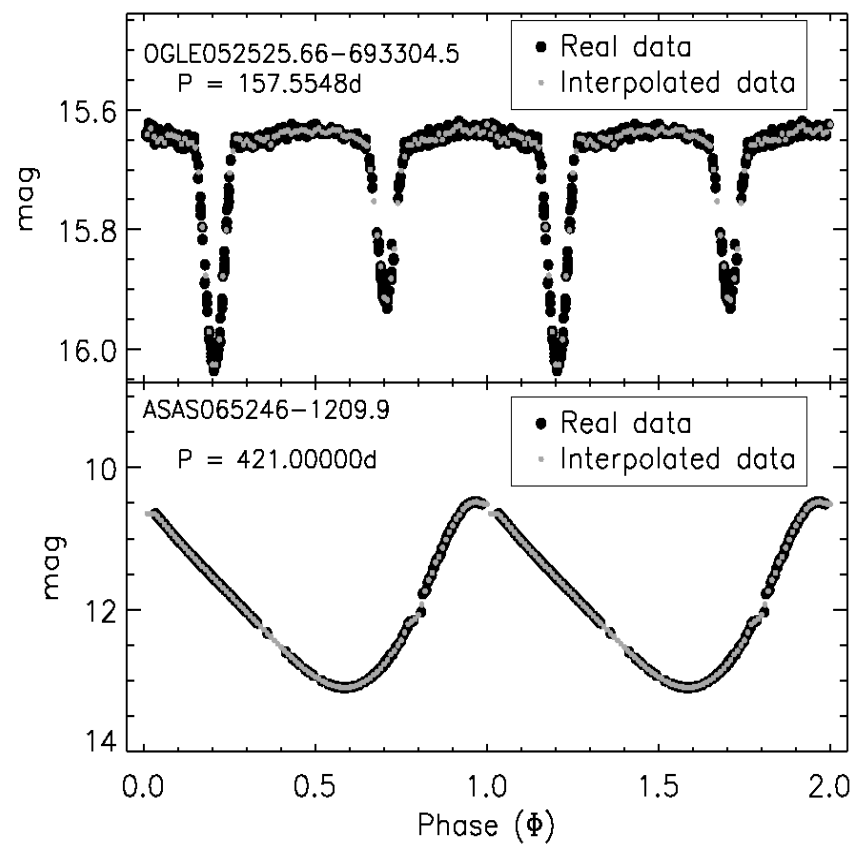

Fig. 3. Examples of interpolation of magnitudes for 100 points. The upper panel shows the light curve with 100 interpolated data points for the OGLE longer period eclipsing binary while the lower panel shows the interpolated data of a long period Mira variable from the ASAS database. The lighter points denote the interpolated data while the bigger black dots represent the original data.

Table 2. The first 10 eigenvectors, their percentage variance and the cumulative percentage of variance of 1829 fundamental mode Cepheids. The input matrix is an $1829 \times 100$ array.

\begin{tabular}{cccc}
\hline \hline PC & Eigenvalue & Percentage & Cum. Percentage \\
\hline 1 & 41.0424 & 41.0424 & 41.0424 \\
2 & 22.8331 & 22.8331 & 63.8755 \\
3 & 11.7668 & 11.7668 & 75.6423 \\
4 & 5.4564 & 5.4564 & 81.0987 \\
5 & 3.6225 & 3.6225 & 84.7212 \\
6 & 2.4477 & 2.4477 & 87.1689 \\
7 & 1.3398 & 1.3398 & 88.5087 \\
8 & 0.7918 & 0.7918 & 89.3005 \\
9 & 0.6435 & 0.6435 & 89.9440 \\
10 & 0.6395 & 0.6395 & 90.5835 \\
\hline
\end{tabular}

relatively smaller number of data points, they did not give any definite conclusions about some of the resonances suggested by Antonello \& Morelli (1996) in the period range $1.38<\log P<$ 1.43. By doing the PCA analysis of the same data as used by Antonello \& Morelli (1996), Kanbur et al. (2002) could not find any feature in that period range. Based on the available light curves covering a wide range of periods, we have plotted $R_{21}$, $R_{31}, \phi_{21}, \phi_{31}$ versus $\log P$ in Fig. 5 . It is evident from the plots that there is a definite structural change in the Fourier coefficients at periods $\log P \sim 1.0$ and 1.5, the latter being close to the period range $1.38<\log P<1.43$ suggested by Antonello \& Morelli (1996). We see that the Fourier decomposition parameters $R_{21}$ and $R_{31}$ decrease until $\log P \sim 1.0$, increase thereafter until $\log P \sim 1.5$ and after that $R_{21}$ and $R_{31}$ fall gradually again until $\log P \sim 2.10$. Similarly in the $\phi_{21}$ and $\phi_{31}$ plane, we see a sharp discontinuity around $\log P \sim 1$. The sharp and the more prominent discontinuity around $\log P \sim 1.0$ is reflected in both $\phi_{21}$ and $\phi_{31}$ plots, whereas the other changes in the light curve 

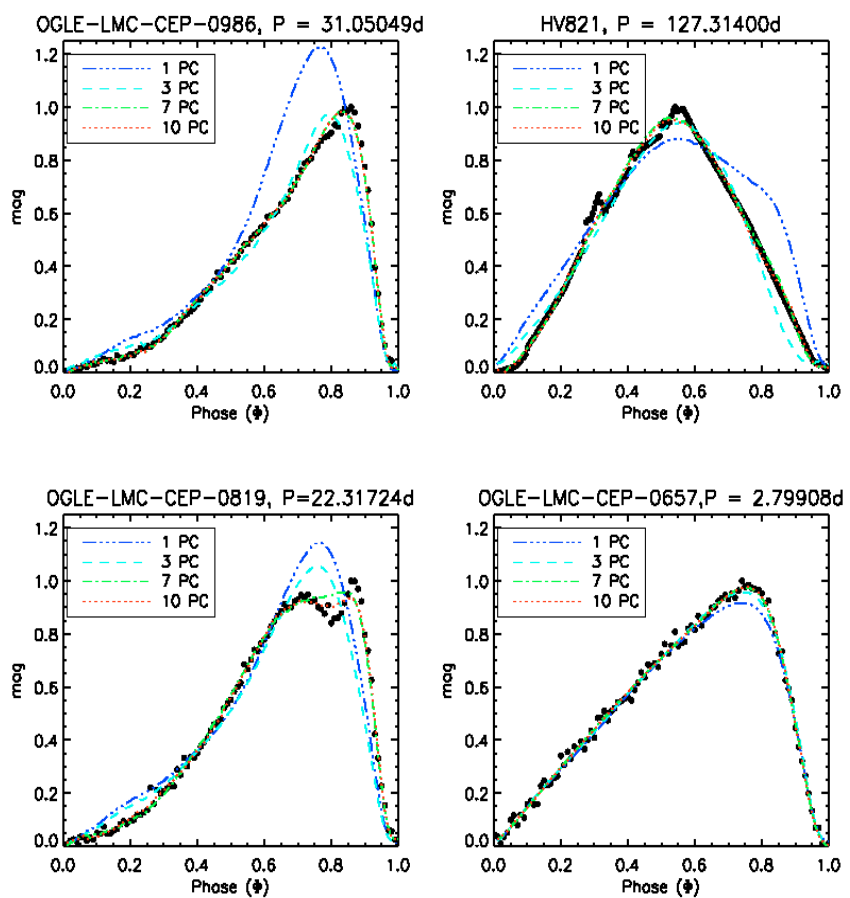

Fig. 4. Reconstruction of FU Cepheid light curves using the first 1, 3, 7 and 10 principal components. The input matrix is an array of 1829 rows (stars) and 100 columns (magnitudes from phase 0 to 1 ). The black dots represent the original 100 interpolated data points normalized to unit magnitude.
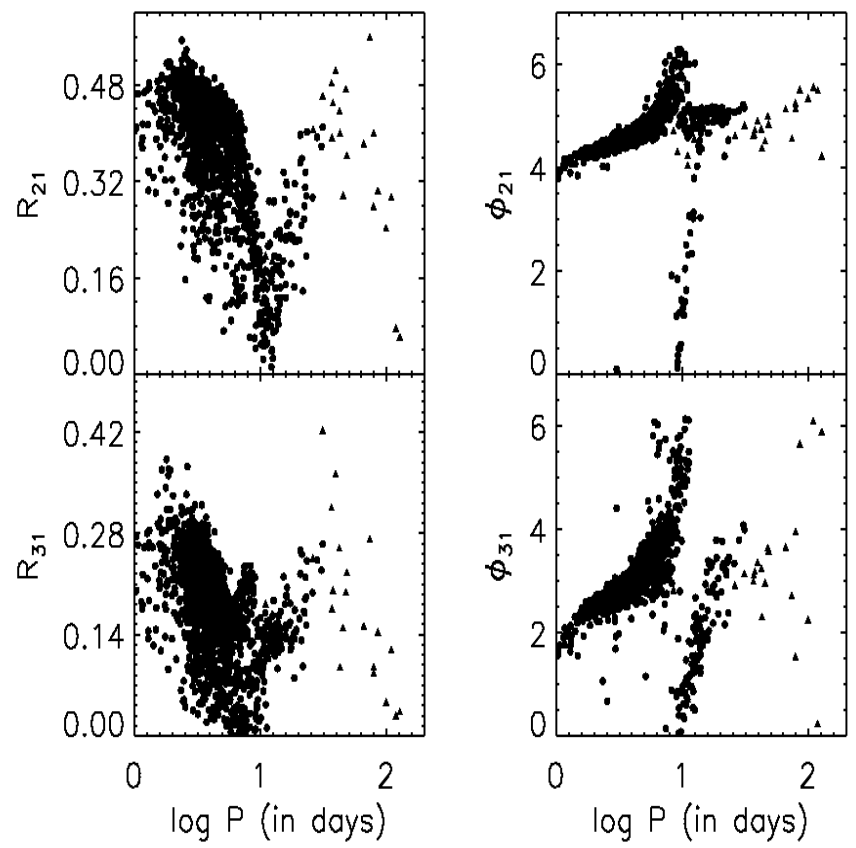

Fig. 5. Fourier parameters $R_{21}, R_{31}, \phi_{21}, \phi_{31}$ as a function of $\log$ (period) for the 1829 FU Cepheids (data set IIA+IIB+IIC, Table 1). The Fourier parameters for the $I$ band stars and $V$ band stars are marked with filled circles and filled triangles respectively.

structures around the period $\log P \sim 1.5$ are visible in all the Fourier parameter plots.

In Fig. 6 we plot the first two PCs and $\mathrm{PC} 1 \times \mathrm{PC} 2(\mathrm{PC} 1 \times 2)$ against $\log P$. For $\mathrm{PC} 1, \mathrm{PC} 2$ and $\mathrm{PC} 1 \times 2$, a discontinuity around $\log P=1.0$ is visible. $\mathrm{PC} 1, \mathrm{PC} 2$ and $\mathrm{PC} 1 \times 2$ clearly show a change around the period $\log P \sim 1.5$. But the

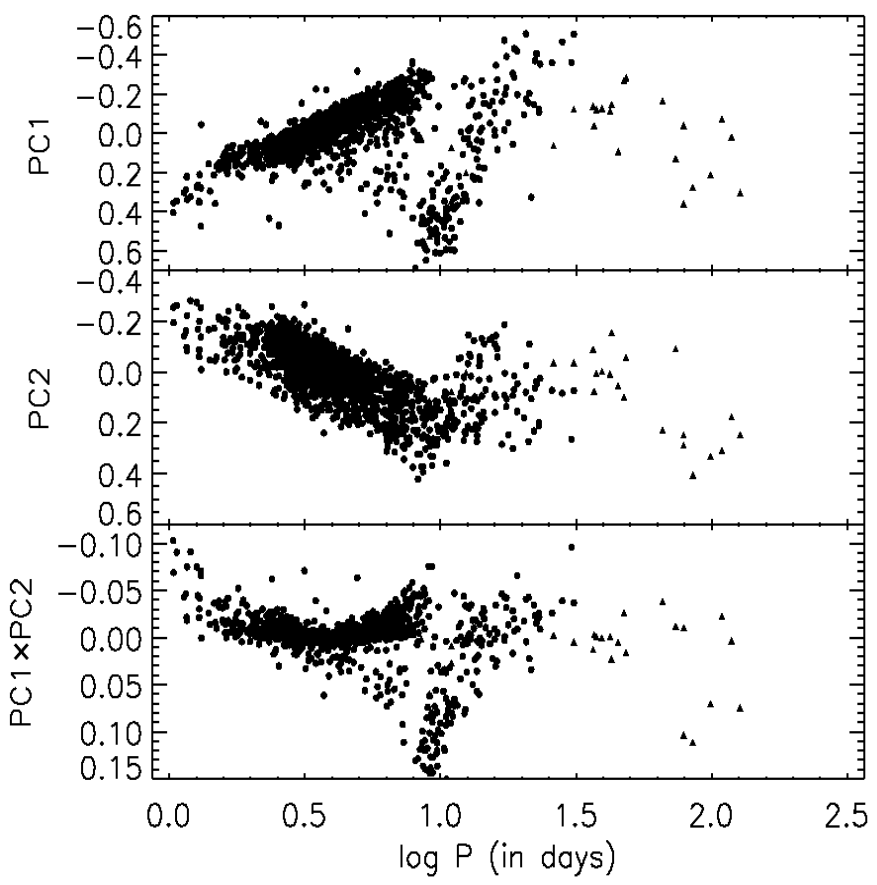

Fig. 6. First two PCs as a function of $\log$ (Period) for the 1829 FU Cepheids (Data set IIA+IIB+IIC, Table 1). The Fourier parameters for the $I$ band stars and $V$ band stars are marked with filled circles and filled triangles respectively.

discontinuity around $\log P \sim 1$ as revealed by the Fourier parameters $\phi_{21}$ and $\phi_{31}$ in Fig. 5 is much more pronounced compared to the PC plots.

Kanbur et al. (2002), using the PCA analysis on the Fourier coefficients, did not find any structure changes in the period range $1.38<\log P<1.43$. Using PCA on a larger light curve data set, we have found that in fact there are structural changes around $\log P \sim 1$ and 1.5 and hence there may exist resonances around these periods. While the resonance around the period $\log P \sim 1$ is well-known, the first two PCs and PC1 $\times 2$ show a change in the light curve structure around $\log P=1.5$. It is difficult to pinpoint the exact location of the change in structure because of fewer stars in the period around $\log P \sim 1.5$. Model calculations are necessary to confirm the existence of this resonance. Further, Antonello \& Poretti (1996) also used a number of data points of the longer period side and found some evidence of a decrease of $R_{21}$ at longer periods around ( $\log P \sim 2$ ). It is difficult to confirm the existence of such a resonance from either FD or PCA although we see some change in trend in the first two PCs around this period. Therefore, although there are changes in the light curve structures around the periods $\log P \sim$ 1.5 and 2.10 days, one cannot confirm the existence of resonances around these periods. Such information about these resonances are generally derived from the combined photometric and spectroscopic observations and radiative hydrodynamical model calculations (Kienzle et al. 1999).

\subsubsection{First overtone (FO) Cepheids}

The light curves of FO Cepheids show a discontinuity in the Fourier phase parameters $\phi_{21}$ and $\phi_{31}$ around a period of $\sim 3.2$ days. This is shown in Fig. 7 for the OGLE data (Data set III) of 1228 FO Cepheids. This feature was interpreted as the signature of 2:1 resonance between the first and fourth overtones (Antonello \& Poretti 1986). This feature was however not 

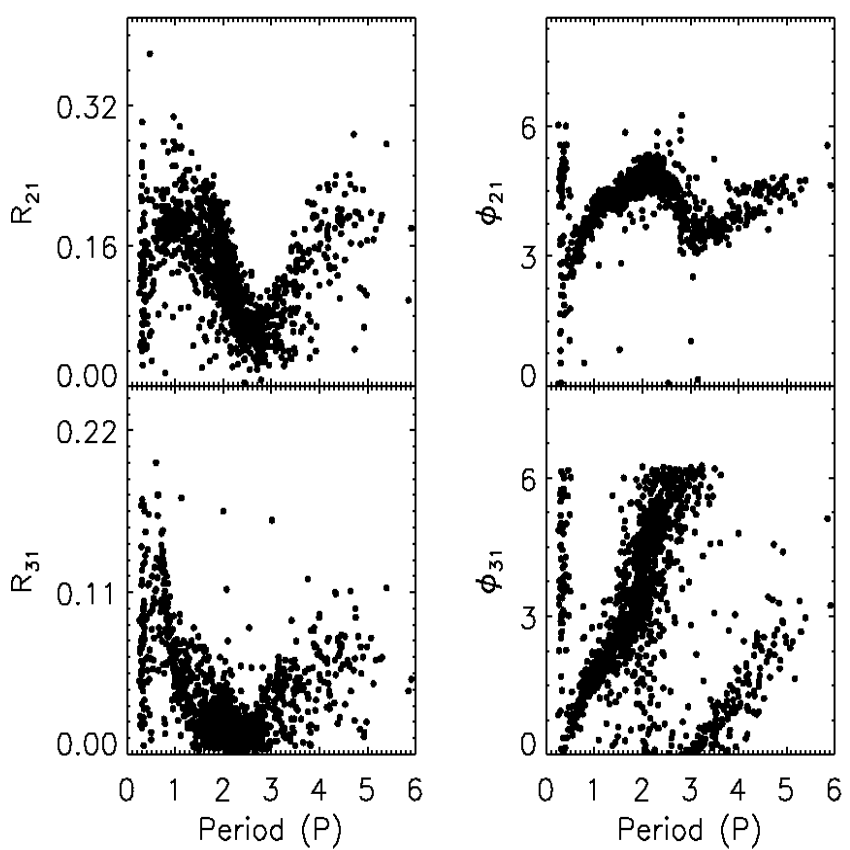

Fig. 7. Fourier parameters $R_{21}, R_{31}, \phi_{21}, \phi_{31}$ as a function of $\log$ (period) for $1228 \mathrm{LMC}$ overtone Cepheids (data set III).

reproduced in the hydrodynamical models and in the Fourier parameters of highly accurate observational radial velocity curves of FO Cepheids (Kienzle et al. 1999). By means of hydrodynamical models for FO Cepheids, Kienzle et al. (1999) have shown that the 3.2 day is not the resonance, the true resonance is at around $4.5 \mathrm{~d}$ and $3.2 \mathrm{~d}$ is not a resonance. On the other hand Buchler et al. (1996) suggested that for a consistent picture on the evolutionary mass-luminosity relations, the FO Cepheid resonance should occur at $P=4.3$ days. Therefore, not all such structures in the photometric Fourier parameters need to be connected to the resonances.

On the other hand, by analyzing the Fourier coefficients of a large number of FO LMC Cepheids in the OGLE III database, Soszyński et al. (2008) found a change in the photometric Fourier parameters around a period of $\sim 0.35 \mathrm{~d}$. The short-period discontinuity at $0.35 \mathrm{~d}$ can be explained by the presence of the $2: 1$ resonance between the first and fifth overtones in stars with masses of about $2.5 M_{\odot}$ (Dziembowski \& Smolec 2009).

In Fig. 7, we plot the Fourier parameters $R_{21}, \phi_{21}, R_{31}, \phi_{31}$ for 1228 LMC FO Cepheids (Data set III in Table 1). The optimal order of the fit to the Fourier method has been found to be 10 . There is a definitive marked structure of discontinuity in the Fourier plots at periods around 0.35 and 3.2 days.

We now try to find out whether our PCA procedure can extract the information about the structure changes. We carry out PCA on a $1228 \times 100$ matrix of 1228 LMC FO Cepheids with $100 I$ band magnitudes corresponding to phase 0 to 1 in steps of 0.01 . Figure 8 shows the plot of first three PCs versus the period. A sharp discontinuity around the shorter period end near 0.35 day is evident in all the PC plots. Also, some change in the light curve structure seems to occur near to 4 days for all the PC plots. There is no change in the light curve structure around 3.2 days in PC2 and PC3 whereas in PC1, there is a change in the light curve shape around a period of $\sim 3.2$ days.

Thus, we see that the Fourier parameters performed better in bringing out the structural changes in FU Cepheids while for FO Cepheids the performance of FD and PCA techniques is similar.

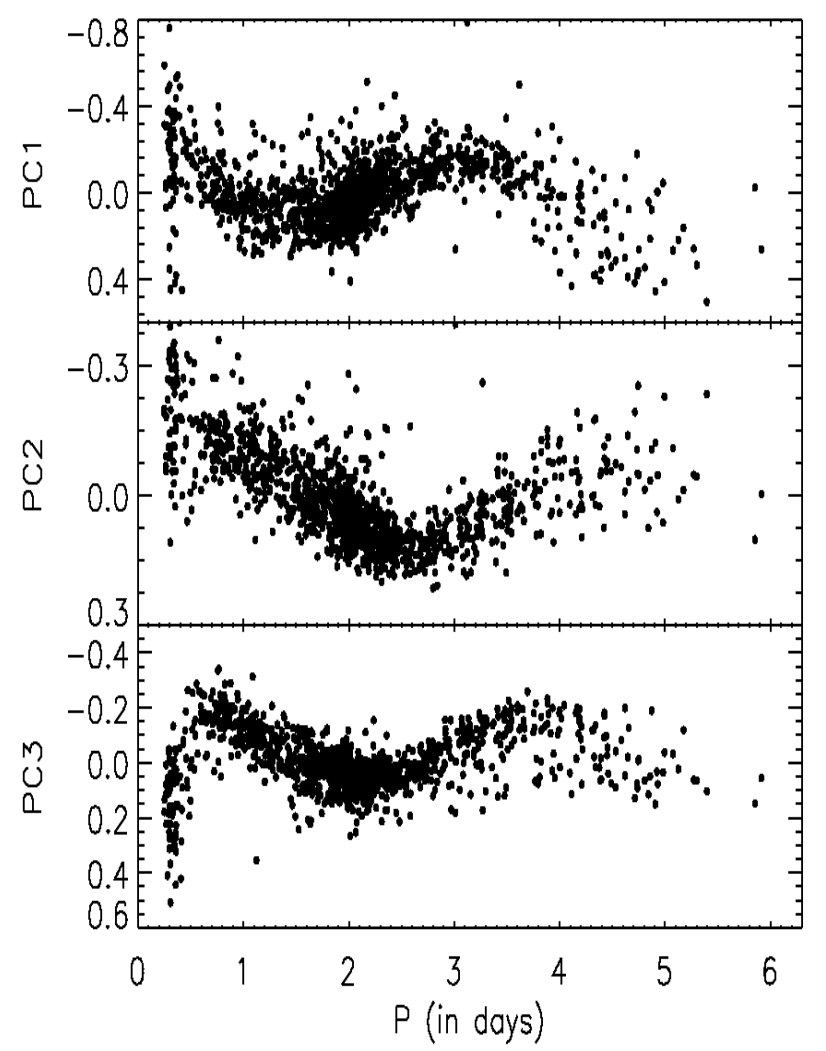

Fig. 8. First three PCs versus period for LMC overtone Cepheids (data set III). The change in the light curve shape as shown in Fig. 7 is also seen from the PC plots. The input matrix is an array of 1228 rows (stars) and 100 columns (magnitudes from phase 0 to 1 ).

\subsubsection{Classification}

We now explore the possibility of classification of different classes of variable stars on the basis of FD \& PCA. We use the Fourier decomposition parameter $R_{21}$ and the first principal component PC1 to classify all the 17606 stars of different variability classes in Table 1. In Fig. 9 we plot the Fourier parameter $R_{21}$ versus $\log P$. As may be seen, the Mira variables form a separate group because of their longer periods and not because of separation in $R_{21}$. However, in the intermediate period range (1 to 100 days), eclipsing binaries have distinct $R_{21}$ values from other classes of variables. Figure 10 shows the plot of $\log R_{21}$ to demonstrate the complete range of $R_{21}$ for 4085 eclipsing binaries. In the short period range there is a considerable overlap between the FO Cepheids and RRc stars. This degeneracy in the Fourier parameter $R_{21}$ in the short period range cannot be lifted and the classification accuracy cannot be improved by further manipulation.

We carry out the PCA on a $17606 \times 100$ matrix of 17606 stars, each star having 100 values of magnitudes in their light curves. We have used the first principal component (PC1) as it contains the maximum variance in this data set. As in the case of FD, the PCA is able to separate the Mira variables and the eclipsing binaries and the separation is more effective in the case of PCA (Fig. 11). The plot of PC1-log $P$ space also shows that although PC1 is able to separate the eclipsing binaries and Mira variables, there is some overlap in the regions dominated by RR Lyraes and Cepheids. In the next step, we choose only the samples of RR Lyraes (RRab \& RRc) and Cepheids (FU \& FO) that could not be separated well by the use of PCA on the whole data set. We now run PCA on 10643 light curves (Data 


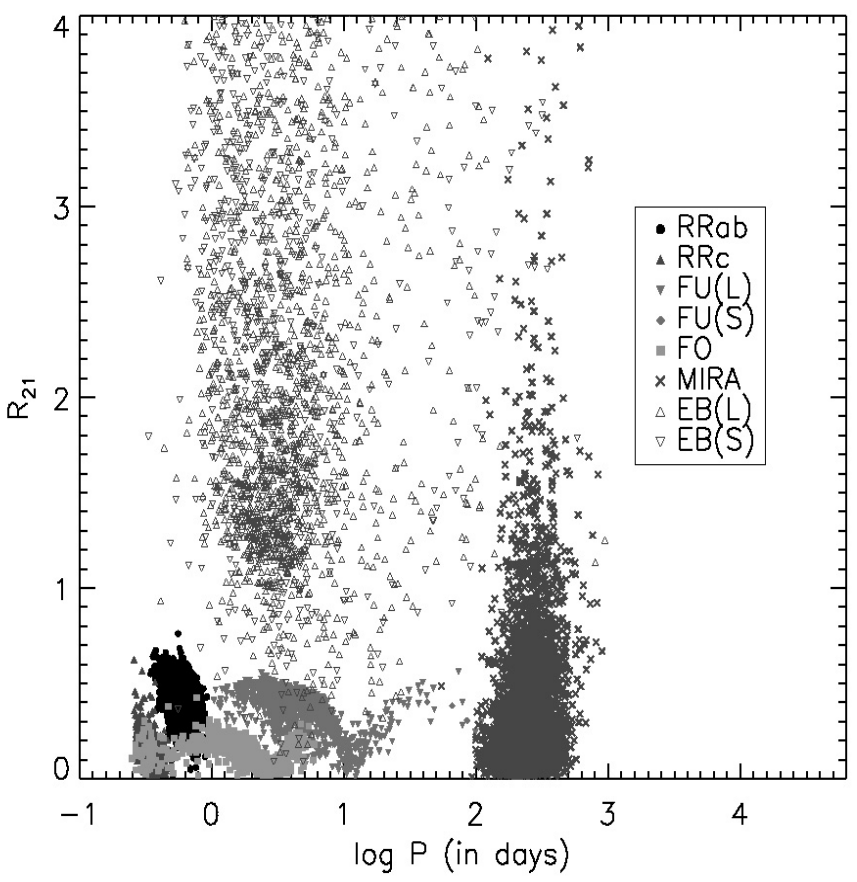

Fig. 9. The classification based on $R_{21}$ obtained from the FD method. $\mathrm{L}$ and $\mathrm{S}$ denote the LMC and SMC objects respectively.

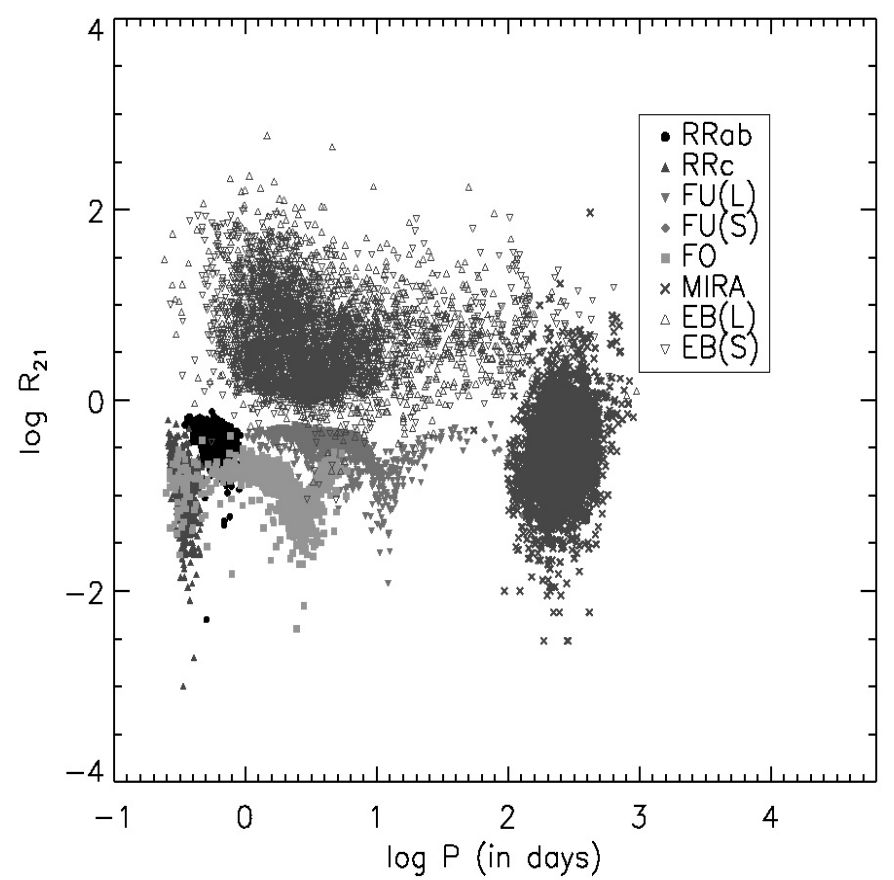

Fig. 10. The classification based on $\log R_{21}$ obtained from the FD method.

set IA + IB + IIA + IIB + IIC + III) of RR Lyraes and Cepheids. The result of PCA on this $10643 \times 100$ array is shown in Fig. 12 . It may be noted that PC1 is able to separate FU Cepheids and RRab stars to a large extent while there is some overlap between $\mathrm{RRc}$ and FO Cepheids in a narrow period range $(0.25-0.5 \mathrm{~d})$. We hope to return to this degeneracy problem in a subsequent study in which we also intend to increase the sample by adding more classes of variables.

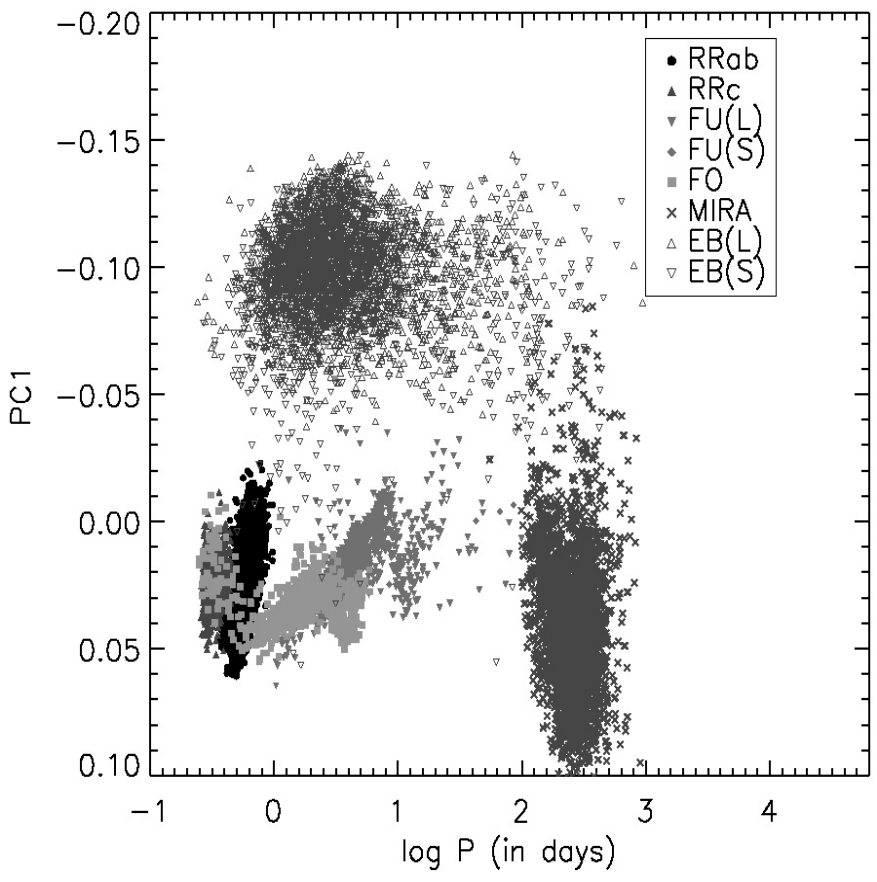

Fig. 11. The classification based on PC1 obtained from PCA of 100 interpolated magnitudes for the phase from 0 to 1 in steps of 0.01 .

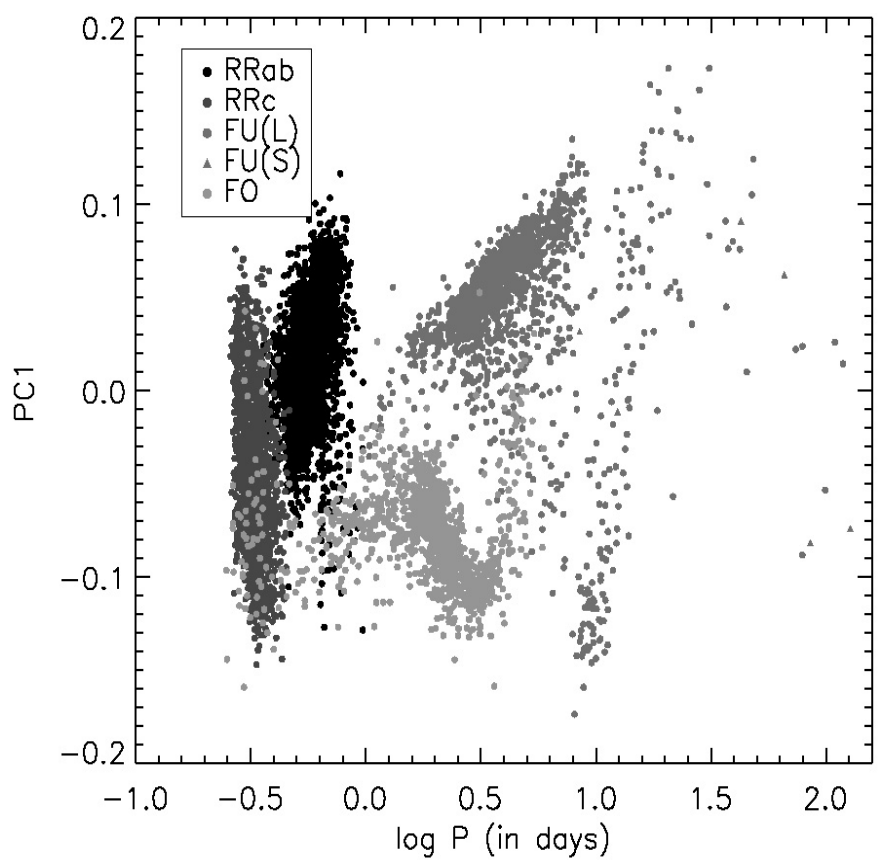

Fig. 12. The classification between RR Lyraes (RRab \& RRc) and Cepheids (FU \& FO) based on PC1.

\section{Conclusions}

Fourier decomposition is a trusted and much applied technique for analyzing the behaviour of light curves of periodic variable stars. It is well suited for studying individual light curves as the Fourier parameters can be easily determined. However, when the purpose is to tag a large number of stars for their variable class using photometric data from large surveys, the technique becomes slow and cumbersome and each light curve has to be fitted individually and then analyzed. The same is true if the aim is to look for resonances in the light curves in an automated way 
for a large class of pulsators. It is, therefore, desirable to look for methods that are reliable, automated and unsupervised and can be applied to the available light curve data directly.

Some attempts have been made in the recent past to use the well known PCA for the light curve analysis, but the major drawback of these studies was that they required the calculation of the Fourier parameters which then went as input to the PCA. This meant that the PCA, which was supposed to replace the Fourier decomposition, in fact relied on it. Also for precise and accurate determination of Fourier parameters, the light curve should have good phase coverage and less noisy data points so that the fit to the light curve is good enough to rely on its parameters. But this is not the case for every light curve data generated from the automated surveys. Sometimes there are gaps and/or outliers in the data. The fitting of such a light curve will give a wrong estimation of the Fourier parameters.

In this paper we have used the original light curve data for computation of the principal components. It involves four simple steps: a) to phase each light curve between 0 to 1 with the respective period in days. b) Interpolation of light curve magnitudes in short steps $(0.01)$ between phase 0 to 1 to obtain 100 points of magnitude for each light curve. c) Normalize the magnitudes between 0 and 1 for each of the light curves and d) to do PCA on the normalized magnitudes of 100 points for all the light curves.

The PCA is then used to analyse the structure of the light curves of classical Cepheids and the results compared with those obtained from the analysis of the Fourier parameters. In addition, the two techniques are compared regarding their ability to classify stars into different variability classes.

We applied the PCA technique to study the structure of light curves of fundamental and first overtone Cepheids. By choosing a large data set of a large range of periods, we have shown that the structure of the fundamental mode Cepheid light curves shows significant changes around the periods $\log P \sim 1$ and 1.5. The resonance around the period $\log P \sim 1$ is well known. The first two PCs also show that the behavior of the light curves changes around the period $\log P \sim 1.5$ which is close to the resonance suggested by Antonello \& Poretti (1996) in the period range $1.38<\log P<1.43$. There is some evidence of a structural change in the light curve shape around the period $\log P \sim$ 2.0 also but this can be confirmed only when longer period data become available. We find that the Fourier parameters performed better in bringing out the discontinuities in FU light curves at periods around $\log P \sim 1$.

For the first overtone LMC Cepheids, we find a discontinuity at a shorter period of $\sim 0.35 \mathrm{~d}$. The first few PCs also show a clear trend of structural changes of the first overtone Cepheids at this short period. For FO Cepheids, the performance of FD and PCA is similar in bringing out the structural changes around a period of 0.35 days. We have been able to find this feature because of the availability of a significant number of light curves towards the shorter period end of the LMC Cepheids in the OGLE database. The PCA technique can easily find similar resonances in the Galactic and SMC first overtone Cepheids as and when there is substantial data available for the short period objects of this class.

We have also demonstrated the ability of PCA and its distinct advantage over the FD method in classifying stars into different variability classes. Although alternative automated methods for variable star classification exist, the PCA based technique can be used as a first step in a hierarchical classification scheme because of its accuracy and efficiency.
The data compression ratio using PCA on the direct light curve data is enormous, a fact that has great relevance when dealing with large databases of the future. Also, we have shown some preliminary results of variable star classification for an ensemble of 17606 stars selected in the present analysis. In a future paper, we will describe the application of the PCA technique to a larger, more diverse database by looking at the classification accuracy and errors.

Acknowledgements. The authors thank the anonymous referee for helpful comments which improved the presentation of the paper and Manabu Yuasa for correcting the first draft. Igor Soszyński is thanked for providing valuable information regarding the OGLE data. S.D. thanks Council of Scientific \& Industrial Research (CSIR), Govt. of India, for a Senior Research Fellowship. H.P.S. thanks CRAL-Observatoire de Lyon for an invited Professorship.

\section{References}

Antonello, E. 1994, A\&A, 282, 835

Antonello, E., \& Morelli, P. L. 1996, A\&A, 314, 541

Antonello, E., \& Poretti, E. 1986, A\&A, 169, 149

Bailer-Jones, C. A. L., Irwin, M., \& von Hippel, T. 1998, MNRAS, 298, 361

Baart, M. L. 1982, IMA J. Num. Analysis, 2, 241

Berdnikov, L. N. 2008, VizieR On-line Data Catalog: II/285, originally published in: Sternberg Astronomical Institute, Moscow

Buchler, J. R., Kollath, Z., Beaulieu, J. P., et al. 1996, ApJ, 462, L83

Chatfield, C., \& Collins, A. J. 1980, introduction to Multivariate Analysis (London: Chapman \& Hall)

Connolly, A. J., Szalay, A. S., Bershady, M. A., Kinney, A. L., \& Calzetti, D. 1995, AJ, 110, 1071

Debosscher, J., Sarro, L. M., Aerts, C., et al. 2007, A\&A, 475, 1159

Dziembowski, W. A., \& Smolec, R. 2009, Acta Astron., 59, 19

Folkes, S. R., Lahav, O., \& Maddox, S. J. 1996, MNRAS, 283, 651

Francis, P. J., Hewett, P. C., Foltz, C. B., \& Chaffee, F. H. 1992, ApJ, 398, 476

Hendry, M. A., Tanvir, N. R., \& Kanbur, S. M. 1999, ASP Conf. Ser. (San Francisco), 167, 192

Jin, H., Kim, S. -L., Lee, C. -U., et al. 2004, AJ, 126, 1847

Kanbur, S. M., \& Mariani, H. 2004, MNRAS, 355, 1361

Kanbur, S. M., Iono, D., Tanvir, N. R., \& Hendry, M. A. 2002, MNRAS, 329, 126

Kienzle, F., Moskalik, P., Bersier, D., et al. 1999, A\&A, 341, 818

Lahav, O., Naim, A., Sodré, L., Jr., \& Storrie-Lombardie, M. C. 1996, MNRAS, 283, 207

Mantegazza, L., \& Poretti, E. 1992, A\&A, 261, 137

Martin, W. L., Warren, P. R., \& Feast, M. W. 1979, MNRAS, 188, 139

Moffett, T. J., Gieren, W. P., Barnes, T. G. III, \& Gomez, M. 1998, ApJS, 117, 135

Moskalik, P., \& Poretti, E. 2003, A\&A, 398, 213

Murtagh, F., \& Heck, A. 1987, Multivariate Data Analysis (Dordrecht: Reidel)

Ngeow, C.-C, Kanbur, S. M., Nikolaev, S., et al. 2003, ApJ, 586, 959

Petersen, J. O. 1986, A\&A, 170, 59

Poretti, E. 2001, A\&A, 371, 986

Press, W., Teukolsky, S., Vetterling, W., \& Flannery, B. 1992, Numerical Recipes in FORTRAN, 2nd edn. (Cambridge Univ. Press)

Sarro, L. M., Debosscher, J., López, M., \& Aerts, C. 2009, A\&A, 494, 739

Schaltenbrand, R., \& Tammann, G. A. 1971, A\&AS, 4, 265

Simon, N. R. 1979, A\&A, 74, 30

Simon, N. R., \& Lee, A. S. 1981, ApJ, 248, 291

Singh, H. P., Gulati, R. K., \& Gupta, R. 1998, MNRAS, 295, 312

Sodré, L. Jr., \& Cuevas, H. 1994, Vistas Astron., 38, 287

Soszyński, I., Udalski, A., Szymanski, M., et al. 2002, Acta Astron., 52, 369

Soszyński, I., Udalski, A., Szymanski, M., et al. 2003, Acta Astron., 53, 93

Soszyński, I., Poleski, R., \& Udalski, A. 2008, Acta Astron., 58, 163

Storrie-Lombardi, M. C., Irwin, M, J., von Hippel, T., \& Storrie-Lombardi, L. J. 1994, Vistas Astron., 38, 331

Tanvir, N. R., Hendry, M. A., Watkins, A., et al. 2005, MNRAS, 363, 749 Udalski, A., Soszyński, I., Szymański, M., et al. 1999, Acta Astron., 49, 437

Udalski, A., Soszyński, I., Szymanski, M. K., et al. 2008, Acta Astron., 58, 89 Wyrzykowski, L., Udalski, A., Kubiak, K., et al. 2003, Acta Astron., 53, 1 Wyrzykowski, L., Udalski, A., Kubiak, K., et al. 2004, Acta Astron., 54, 1 